\title{
Multimodal ultrasound investigation (grey scale, Doppler and 2D-SWE) of salivary and lacrimal glands in healthy people and patients with diabetes mellitus and/or obesity, with or without sialosis
}

\author{
Maria Bădărînză*1, Oana Șerban*1, Lavinia Maghear1, Corina Bocșa1, Mihaela Micu², \\ Mihai Dumitru Porojan', Bogdan Augustin Chiș ${ }^{1}$, Adriana Albu${ }^{1}$, Daniela Fodor ${ }^{1}$ \\ * The authors shared the first authorship
}

${ }^{1} 2^{\text {nd }}$ Internal Medicine Department, "Iuliu Hatieganu" University of Medicine and Pharmacy ${ }^{2}$ Rheumatology Division, Rehabilitation Clinical Hospital, Cluj-Napoca, Romania

\begin{abstract}
Aim: To evaluate the ultrasound (US) modifications [grey scale, Doppler, 2D-share wave elastography (2D-SWE)] of salivary (parotid and submandibular) and lacrimal glands in healthy people and patients with diabetes mellitus and/or obesity, with or without sialosis. Material and methods: We evaluated 170 patients (1020 glands, 1700 grey scale and Doppler images), split in two groups (group 1- healthy people, group 2- obese and/or diabetes patients, with or without sialosis). For each patient we assessed the parotid, submandibular and lacrimal glands in grey scale US (echogenicity, homogeneity, glandular contour, posterior border, lymph nodes), color Doppler US and 2D-SWE. All images were analyzed by two examiners blinded to each other or to patients. Results: The interobserver agreement was strong or moderate for all parameters. In group 2, the salivary glands had increased echogenicity, homogeneous aspect and invisible posterior border (all $\mathrm{p}<0.001$ ). There was no significant variation of elasticity modulus in the groups analyzed ( $5.46 \pm 1.57$ vs $5.67 \pm 1.81$ in parotid, $8.63 \pm 1.84$ vs $8.55 \pm 1.94$ in submandibular and $9.47 \pm 2.1$ vs $9.53 \pm 2.23$ in lacrimal glands, all $\mathrm{p}>0.05$ ) or according to the body mass index (BMI), sex, patient age, the aspect in grey scale/Doppler US or the presence of sialosis (all p>0.05). Conclusion: The main US differences between healthy people and patients with diabetes mellitus and/or obesity are suggested by the echogenicity, homogeneity, posterior border and the size of glandular area. No significant differences of elasticity modulus were found between the analyzed groups or related to BMI, sex, patient age or other grey scale/Doppler US items analyzed.
\end{abstract}

Keywords: salivary glands; lacrimal glands; ultrasound; 2D-SWE; sialosis

\section{Introduction}

Ultrasound (US) is considered to be the first-line imaging technique in investigating the salivary glands [1] Only in selected cases (suspected malignant tumors or

Received 16.08.2019 Accepted 24.08.2019

Med Ultrason

2019, Vol. 21, No 3, 257-264

Corresponding author: Daniela Fodor, MD, PhD

$2^{\text {nd }}$ Internal Medicine Department

"Iuliu Hatieganu" University of Medicine and

Pharmacy, Cluj-Napoca, Romania

2-4 Clinicilor street,

400006 Cluj Napoca, Romania

E-mail: dfodor@umfcluj.ro

Phone number: +40264597852 lymph nodes, the presence of masses in the deeper lobe of parotid gland or under the mandible) computed tomography (CT) or magnetic resonance imaging (MRI) are necessary [2]. The normal salivary gland has a regular contour, homogeneous US aspect, intermediate echogenicity and lymph nodes. The deep parotid lobe may be difficult to be completely examined by US, especially due to the acoustic shadow of the mandibular ramus [3].

Elastography was recently introduced in the evaluation of the salivary glands' stiffness [4-6], strain elastography (SE) and acoustic radiation force impulse (ARFI) techniques being the first ones used to characterize the tumoral pathology or the diffuse inflammatory diseases. Recently, share wave elastography (SWE), a real-time, quantifiable and user-independent method, gains atten- 
tion for salivary glands investigation. The elasticity is expressed as elasticity modulus (or Young's modulus), measured in kiloPascals $(\mathrm{kPa})$ or as shear wave velocity, measured in meters/second $(\mathrm{m} / \mathrm{s})$.

The applicability of SWE in evaluating liver fibrosis, breast or thyroid pathology, etc. was already demonstrated [7-9], but for salivary gland the utility of the method is still unclear, mainly due to insufficient reported data [10, 11] and lack of standardization of the normal values. No differences between sexes and increase in parotid stiffness with age or body mass index (BMI) were found [12, 13] and no specific data about the parotid modifications in the presence of diabetes mellitus was published. For pediatric population, values of normal parotid gland elasticity using SWE were recently published [14], but more measurements are needed.

Lacrimal gland US is less used in clinical practice. The superficial location in superolateral part of the orbit permits the evaluation in good conditions, the gland being recognized as a small hypoechoic, homogenous area [15]. No data on the elastography of the lacrimal glands were published.

Apart from the inflammatory (including autoimmune) diffuse diseases of the salivary glands, a particular pathology in which the glands are diffusely modified is sialosis (or sialoadenosis) a chronic, diffuse, non-inflammatory, non-neoplastic disease, associated with bilateral painless swollen parotid glands [16]. The most common causes are chronic alcoholism, diabetes and obesity, but there are other incriminated rare conditions, such as malnutrition, bulimia or drugs [16]. Histological evaluation found contradictory results about acinar enlargement and fatty infiltration [17]. The diagnosis is clinically suspected (enlargements of salivary glands) and is completed with US evaluation or, rarely, using biopsy to rule out other diagnosis [18]. US examination shows homogenous, hyperechoic and enlarged salivary glands [19]. To our knowledge, there are no available data regarding the salivary and lacrimal glands elastographic evaluation in patients with sialosis.

In our experience, we frequently found modifications during the routine grey scale US examination of salivary gland parenchyma, modifications that were difficult to interpret and correlate with the patient's pathology.

The aim of our study was to evaluate the salivary and lacrimal glands in healthy people and patients with diabetes mellitus and/or obesity (with or without sialosis) using multimodal US (grey scale, color Doppler, elastography).

\section{Material and methods}

\section{Patients}

We conducted a prospective cross-sectional study, from April to July 2018, on 221 consecutive patients referred to our day-hospital clinic. Patients with previous radiation of the neck, hepatitis $\mathrm{C}$, acquired immunodeficiency disease, sarcoidosis, autoimmune diseases or with recent pathology of the salivary and lacrimal glands were excluded (51 patients). Finally, a total number of 170 patients were included. Demographic data (age, sex, weight, high) had been collected and clinical examination of the salivary glands and biological tests (cholesterol, triglyceride, glycemia, glycosylated hemoglobin)

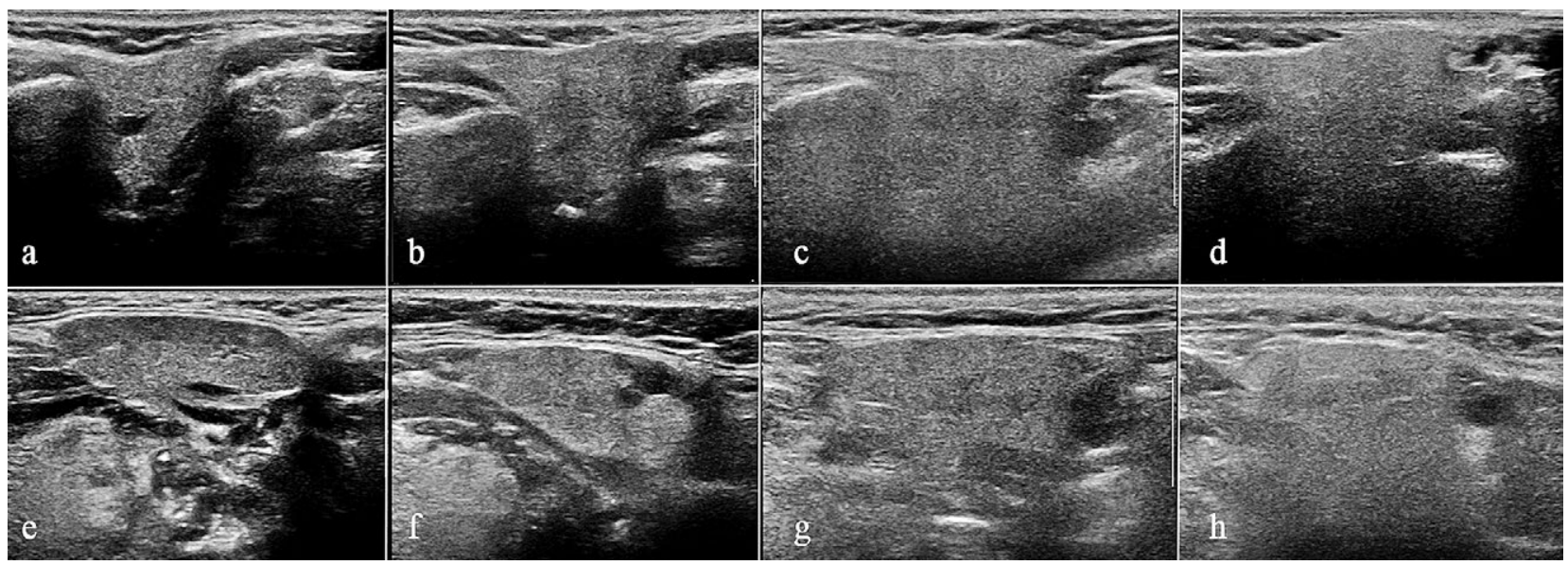

Fig 1. Grey scale ultrasound of the parotid and submandibular glands. The echogenicity scale (atlas) of homogenous parotid glands (a-d): a - normal with clear posterior border, b - slightly increased with visible posterior border, c - moderate increased with invisible posterior border, $\mathrm{d}$ - highly increased and no visible posterior border; The echogenicity scale (atlas) of submandibular glands (e-h): e - normal, f - slightly increased, g - moderate increased with partial visible posterior border, $\mathrm{h}$ - highly increased with invisible posterior border 
had been performed. Body mass index (BMI) had been calculated $\left(\mathrm{kg} / \mathrm{m}^{2}\right)$. Definitions of obesity, diabetes and hyperlipemia were in agreement with international standards [20-22]. Each patient signed an informed consent and the study was approved by the Ethics Committee of University (nr. 166/2.04.2018).

\section{US and SWE examination}

The US and elastography were realized with Supersonic Imagine AixplorerUltimate machine (SuperSonic Imagine, Aix-en-Provence, France) that has the SuperSonic Imagine's UltraFast ${ }^{\mathrm{TM}}$ software technology, and using the multi-frequency linear transducer (SL18-5 $\mathrm{MHz}$ ).

In order to ensure homogeneous evaluation, prior to the study, an atlas with representative images of each US parameters evaluated was created (consensus D.F., M.M. and C.B., all with more than 15 years of experience in US) and was used as reference (fig 1 and 2).

The patients were examined in supine position, with the head turned to the opposite side for parotid and lacrimal glands examination and the head slightly extended for submandibular glands examination. The patients were asked to breath normally and not to swallow during the procedure. The glands were assessed in longitudinal and transvers scans, but the measurements were performed in transverse for parotid (at mandibular angle level) and for lacrimal glands, and in longitudinal for submandibular glands (parallel to mandible). To establish the area of salivary glands we used two methods: 1) by drawing the surface area (measured surface area) and 2) by using the formula (length $\mathrm{x}$ width)/2 (calculated surface area) as previously described [23]. To better evaluate the area of the salivary glands, the US focus was adjusted for deep structures. The following parameters were evaluated: echogenicity ( 0 - normal, 1 - slightly increased, 2 - moderate increased, 3 - highly increased), homogeneity ( 0 - homogeneous, 1 - inhomogeneous), glandular contour (0 - regular, 1 - irregular), posterior border (0 - visible, 1 - invisible), lymph nodes ( 0 - absent, 1 - present) and parenchyma vascularization ( 0 - normal, 1 - increased). The normal echogenicity was considered to be similar to the normal thyroid gland. We used only two parameters for the evaluation of lacrimal gland: echogenicity $(0$ - normal, 1 - increased) and homogeneity ( 0 - homogeneous, 1 - inhomogeneous). For color Doppler US pulse repetition frequency was $700 \mathrm{~Hz}$ and color gain was adjusted dynamically to maximize visualization of the arteries while minimizing the noise.

For 2D-SWE evaluation, a sufficient quantity of ultrasound gel was used and no pressure was applied on the examined tissues. Because all the presetting for musculoskeletal system returned with improper elastography

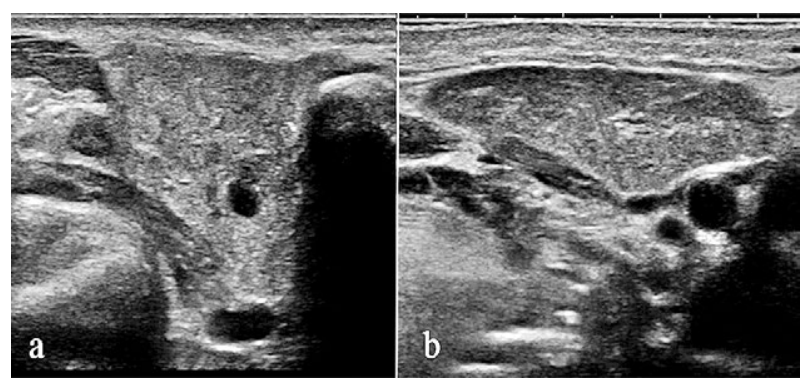

Fig 2. Grey scale ultrasound of parotid (a) and submandibular (b) glands showing inhomogeneous parenchyma

boxes, we used the presetting for thyroid examination. We guided the 2D-SWE examination considering the general rules for liver evaluation [24]. For measurements, we used the elasticity modulus (Young's modulus E). A measurement was considered to be reliable if the stability index was $>90 \%$. For salivary glands, a 3-mm diameter Q-box was placed in the superficial parenchyma in a representative homogeneous region (without vessels or lymph nodes) at $1.5 \mathrm{~cm}$ from the glandular capsule. Three consecutive measurements were made at the same depth and, using a multi Q-box function, the machine returned mean and median values in $\mathrm{kPa}$. For the lacrimal glands, a 2-mm diameter Q-box was placed in the middle of the lacrimal gland (fig 3). We acknowledged the presence of vertical striped or push artefact [25] and all measurements were performed between the artifactual stripes.

All the images were saved and stocked. The images were analyzed by 2 examiners with 3 and 4-year experience (M.B. and O.S.) in salivary glands US. All the disagreements were solved by a third person (DF) with 20 years of experience in salivary gland US. Totally, 1700 grey scale and color Doppler images (10 images for each patient) were analyzed by completing a standardized worksheet with all analyzed parameters.

\section{Statistical analysis}

The distribution of the variables was evaluated using the Kolmogorov-Smirnov test. A descriptive analysis was performed and continuous data was reported as mean and standard deviation. Continuous variables were compared between groups using 2 independent sample t-test for normal distributed variables and Man-Whitney $\mathrm{U}$ for 2 samples in non-normal distributed variables. The comparison of continuous variables between groups with more than 2 levels was performed using Kruskal-Wallis test in non-normal distributed variables. The association between categorical variables was assessed using Chisquare or Fisher's Exact test. Spearman rank correlation was used to correlate non-normal distributed continuous variables. The inter-observer agreement was calcu- 


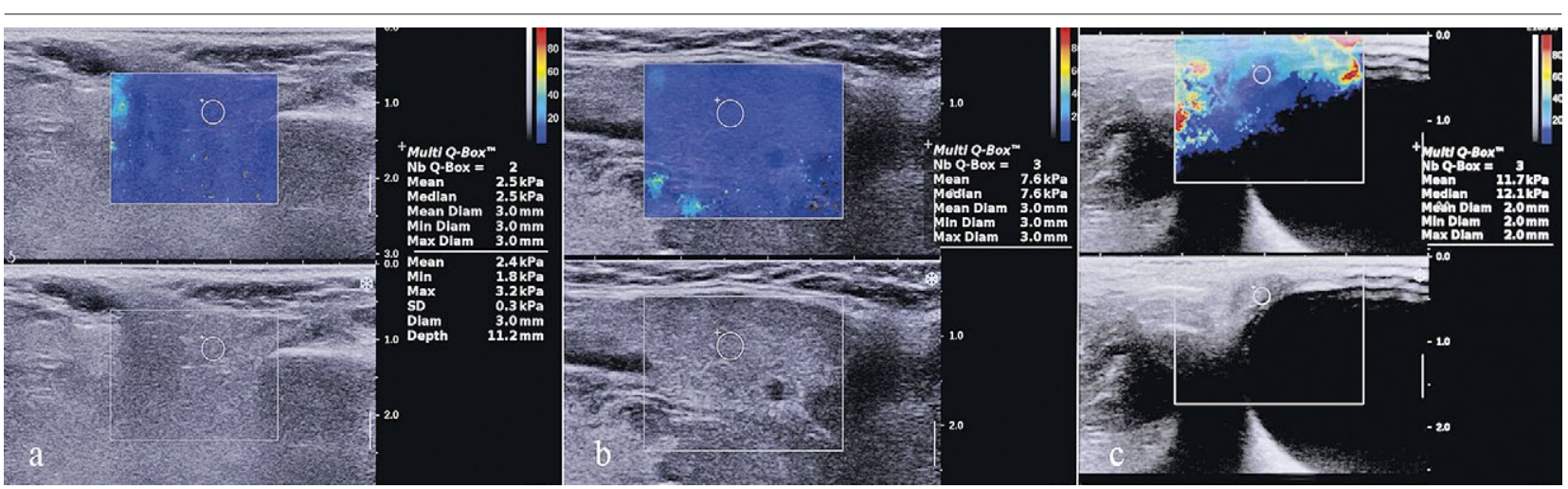

Fig 3. Exemplification of the 2D-SWE measurements in parotid (a), submandibular (b) and lacrimal (c) glands

lated using Cohen's kappa for dichotomous variables and weighted kappa for ordinal variables with more than two categories. P-value $<0.05$ was considered statistically significant. The analysis was performed using IBM SPSS Statistics v.23 and Microsoft Excel 2017 for Office 365.

\section{Results}

A total number of 170 patients (123 women, mean age $59.4 \pm 11.9$ years) were included and they were split in two groups: group 1 - healthy people and group 2 patients with diabetes and/or obesity. The demographic data, area of the glands and the results of SWE measurements are detailed in Table I. Since we found significant differences between the two modalities of area measurement, we used the traced areas for subsequent analysis. No statistical difference between the groups regarding the 2D-SWE measurements was found (all $\mathrm{p}>0.05$ ).

The inter-observer agreement was strong for glands contour, posterior border and vascularization in salivary glands ( $\mathrm{k} 0.93,0.95$, and 0.95 , respectively). For the other parameters (echogenicity, homogeneity, lymph nodes) the agreement was moderate ( $\mathrm{k}$ 0.61-0.66). Considering only 2 groups of echogenicity (normal and slightly increased versus moderate and highly increased), the interobserver agreement dramatically improved from moderate to strong, for both parotid and submandibular glands (k 0.83 and 0.85 ).

Table I. Descriptive analysis of demographic data, glands area and elasticity index in healthy people (group 1) and patients with diabetes and/or obesity (group 2)

\begin{tabular}{|c|c|c|c|}
\hline & Group $1 \quad(N=79)$ & Group $2 \quad(N=91)$ & p-value \\
\hline Age & $44.78 \pm 17.5(22 ; 82)$ & $60.29 \pm 12.99(24 ; 89)$ & $<0.001$ \\
\hline Sex (female) & 69.6 & 74.7 & \\
\hline BMI & $23.82 \pm 3.32(17.96 ; 29.99)$ & $33.12 \pm 5.99(19.43 ; 60.14)$ & $<0.001$ \\
\hline Diabetes & 0 & 37.4 & \\
\hline Dyslipidemia & 12.7 & 56 & \\
\hline \multicolumn{4}{|l|}{ Parotid glands } \\
\hline Measured surface area $\left(\mathrm{cm}^{2}\right)$ & $4.63 \pm 1.39(2.43 ; 9.46)$ & $6.99 \pm 2.4(1.78 ; 13.74)$ & $<0.001$ \\
\hline Calculated surface area $\left(\mathrm{cm}^{2}\right)$ & $3.42 \pm 1.12(1.42 ; 6.25)$ & $4.52 \pm 1.18(1.9 ; 7.6)$ & $<0.001$ \\
\hline $\operatorname{SWE}(\mathrm{kPa})$ & $5.46 \pm 1.57(2.2 ; 10.9)$ & $5.67 \pm 1.81(2.6 ; 12.3)$ & 0.49 \\
\hline \multicolumn{4}{|l|}{ Submandibular glands } \\
\hline Traced surface area $\left(\mathrm{cm}^{2}\right)$ & $2.9 \pm 0.69(1.46 ; 5.65)$ & $3.37 \pm 0.99(1.61 ; 6.92)$ & $<0.001$ \\
\hline Calculated surface area $\left(\mathrm{cm}^{2}\right)$ & $1.77 \pm 0.49(0.73 ; 3.61)$ & $1.99 \pm 0.64(0.91 ; 4.62)$ & $<0.001$ \\
\hline SWE (kPa) & $8.63 \pm 1.84(5.1 ; 13.4)$ & $8.55 \pm 1.94(4.7 ; 13.2)$ & 0.56 \\
\hline \multicolumn{4}{|l|}{ Lacrimal glands } \\
\hline Traced surface area $\left(\mathrm{cm}^{2}\right)$ & $0.04 \pm 0.01(0.02 ; 0.09)$ & $0.04 \pm 0.01(0.01 ; 0.17)$ & 0.83 \\
\hline Calculated surface area $\left(\mathrm{cm}^{2}\right)$ & $0.03 \pm 0.02(0.01 ; 0.26)$ & $0.03 \pm 0.01(0.009 ; 0.1)$ & 0.53 \\
\hline SWE (kPa) & $9.47 \pm 2.1(4.8 ; 15.9)$ & $9.53 \pm 2.23(3.8 ; 15.5)$ & 0.80 \\
\hline
\end{tabular}

The results are expressed as mean \pm standard deviation (SD) and minimum-maximum (min;max) or percent (\%); N, number of patients; BMI, body mass index; SWE, share wave elastography; kPa, kilopascals 
Comparing the US and SWE parameters, no significant differences were noted between left and right glands (all $\mathrm{p}>0.05$ ). Significant differences between sexes were found for glands area $(p<0.001)$ but not for elasticity modulus. According to the age groups (20-40 years, 40-60 years, $>60$ years) the area of parotid and lacrimal glands was found to increase with the age $(\mathrm{p}<0.01)$ but elasticity modulus had nonsignificant variations $(\mathrm{p}>0.05)$. A positive statistical correlation was found between salivary glands area and BMI (as BMI increases, the area becomes larger), but not for elasticity modulus (fig 4).

Comparing the two groups (healthy people and patients with diabetes and/or obesity), we found that in most of the diabetes and/or obese patients the salivary glands had increased echogenicity, homogeneous aspect and invisible posterior border (all $\mathrm{p}<0.001$ ). The mean values of the elasticity modulus had no significant difference between groups (Table II). Also, for lacrimal glands there was no difference of mean elasticity modulus between the groups $(9.47 \pm 2.1$ versus $9.53 \pm 2.23, \mathrm{p}=0.807)$. No correlation between glycemia and glycosylated hemoglobin and US parameters was established in diabetes patients $(\mathrm{p}>0.05)$. Also, in obese patients no correlation was found between US findings and cholesterol or triglyceride levels $(\mathrm{p}>0.05)$.

We found 18 patients $(19.8 \%)$ in group 2 with enlarged clinical parotid glands (sialosis subgroup). US confirmed the increased dimensions of the parotid, found in all cases moderate or highly increase in echogenicity and invisible posterior border, but no significant difference in elasticity modulus was detected when compared to non-sialosis subgroup.

\section{Discussions}

The main conclusion of our study is the lack of differences between elastic modulus of salivary and lacrimal glands, in normal versus diabetes and/or obese subjects, irrespective of sex, age, BMI or glands aspect in grey scale or color Doppler US. Moreover, we found no significant differences in elastic modulus between sialosis and non-sialosis patients.

These results were surprising as we started the study by assuming that diabetes and obese patients have well known elastographic liver modifications, due to non-alcoholic fatty liver disease (NAFD), pathology that can be evaluated by elastography [26]. Parotid modifications in grey scale US in these categories of patients were described [27]. Lack of fibrosis despite other histopathological modifications [17] probably explains our results. The normal parotid elasticity in obese and/or diabetes patients is of major importance - any elastic modulus modification of the salivary and lacrimal glands has to be carefully investigated, as its significance is most probably related to a salivary gland disease itself and is not a response to the metabolic syndrome. Due to lack of the convex probe, we did not investigate the elastic modulus of the liver, so we can draw only indirect conclusions about the presence of NAFD in our patients.

It is difficult to compare our SWE results with results from other studies, as there are important differences between published studies. The main differences are related to the machines used: Toshiba Medical Systems [14], Siemens Acuson S1000 [5] or Philips iU22 [10]. In studies in which Supersonic Imagine Aixplorer machine was used, the authors did not specify the type of Super-
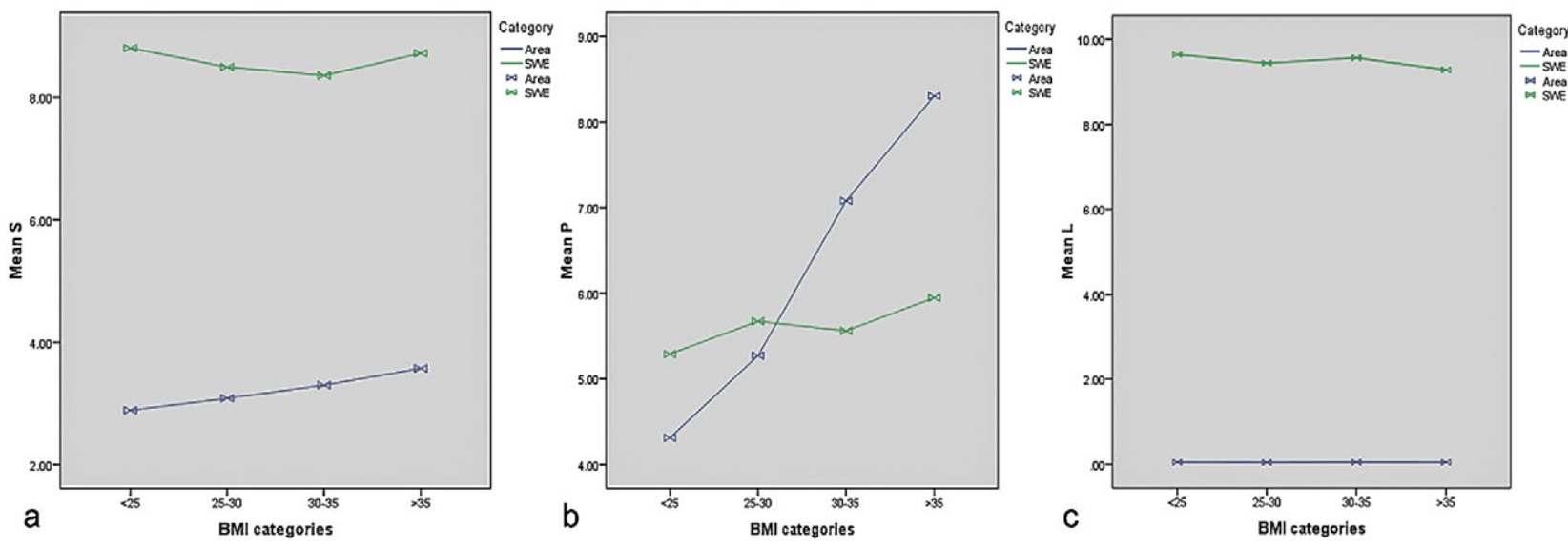

Fig 4. The mean area and 2D-SWE elastic modulus of parotid, submandibular and lacrimal gland according to body mass index (BMI) categories $\left(<25,25-30,30-35,>35 \mathrm{~kg} / \mathrm{m}^{2}\right)$. The mean area increases as BMI increases in submandibular (a - blue line), parotid (b - blue line) glands, but SWE values are constant for both salivary glands ( $a$ and $b$ - green line). In lacrimal glands (c) there are no variations of gland area (blue line) or elastic modulus (green line) according to BMI categories 


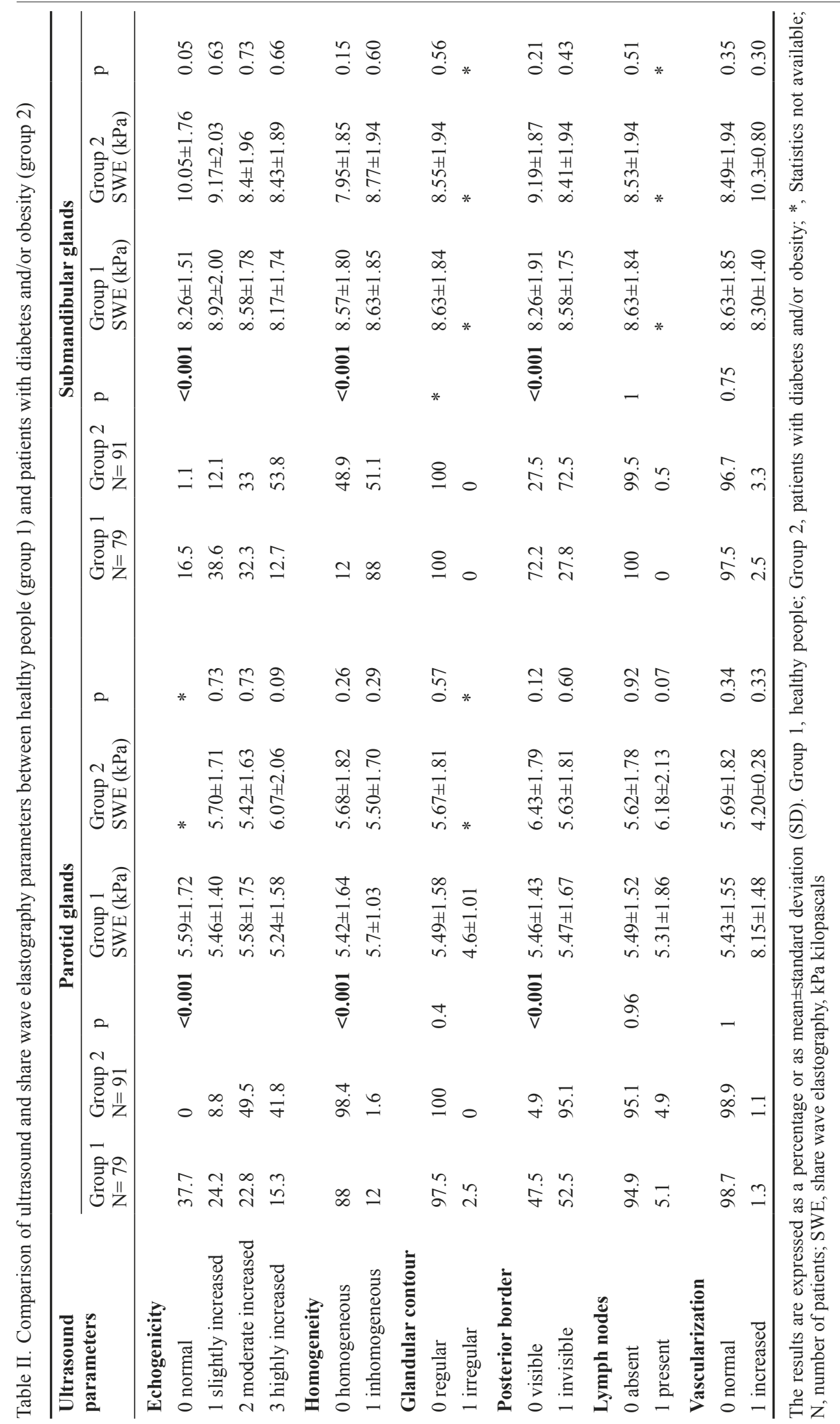


sonic Imagine Aixplorer platform $[12,13,27,28]$ and the frequencies of the transducers used were different: 5-15 $\mathrm{MHz}[28,29], 6-13 \mathrm{MHz}$ [12] or 4-15 [13,27]. We used the Supersonic Imagine AixplorerUltimate machine with 5-18 MHz transducer. As there are no published comparative studies between all these systems/platforms and transducers, no general conclusion can be reached. We also found major differences concerning the protocol of SWE examination: different depths for SWE data collecting, even in the same US scan, the dimensions of the Q-box, the lack of data about the vertical striped artefact or the presetting program used. All these overline the need for supplementary studies, in order to establish interchangeable normal values.

The elastic modulus values obtained in salivary and lacrimal glands (in submandibular gland higher compared to parotid gland, probably related to the different anatomical structures that can be found in these glands) can be used for further comparative studies. We are not sure about the clinical relevance of the data obtained from lacrimal glands examination, as no results were published about pathological findings on elastography.

For the first time we compiled an atlas of grey scale US modifications in these glands in healthy people and, by extension, in diabetic and obese patients. The development of this atlas involved the consensus between 3 experts, after the analysis of more than 1000 images.

Despite the opinion that US is an operator-dependent technique, generally we obtained strong inter-observer agreement for the majority of the studied items. The most challenging was the appreciation of echogenicity when we used the 4 grades classification. High differences in appreciation appeared between grade 0 (normal) and grade 1 (slightly increased) and also between grade 2 (moderate) and grade 3 (highly increased). As grade 0 and 1 were frequent encountered in normal subjects, and 2 and 3 in diabetes/obese patients, we concluded that grade $0-1$ corresponds to normal variation and grade 2-3 is rather pathological (the classification in two classes of echogenicity raised the agreement to strong).

Even if the salivary glands in healthy people are described as homogeneous [19], we found a part of the glands to be rather inhomogeneous. The presence of multiple vessels in submandibular glands and the variability of longitudinal section could explain in part this inhomogeneous appearance. The parenchyma of salivary glands in diabetes and/or obese patients was homogeneous in the majority of cases, probably due to the increased fatty deposition.

We found that the sectional area of parotid and submandibular gland increases with BMI, contrary to the conclusions of Herman et al [13], probably related to the low number of obese patients enrolled in their study.
Also, we found that the area of parotid and lacrimal gland (but not submandibular gland) increased with age, as opposed to the observation published by Dost et al [30], probably related to the lower mean age of the study group (45 years compared to 59.4 years in our study).

Our study has several limitations. First limitation is related to the lack of comparison of the US findings to the biopsy specimens of parotid and submandibular gland. Due to ethic considerations the biopsy was considered an invasive method. Second, no comparison between US and other imaging technique (especially MRI elastography) was realized. The comparison would be of great importance in assessing the gland fibrosis and fatty infiltration and to compare with US findings. Also, the glandular area of salivary glands in patients with invisible posterior border was sometimes difficult to be assessed (and this can be a source of biases) and the comparison with other imaging techniques would have been useful. The same situation was encountered in several cases in which the lacrimal gland was barely visible from orbital fat. Third, we were not aware of the time period between the last mastication and the US evaluation and we did not verify if this could have had any influence on the SWE results. Residual confounding by factors which we failed to control could have also influenced our results.

For future work, it would be interesting to correlate the US data of parotid glands with diabetes characteristics of patients (year onset, the presence of diabetes complications, controlled or uncontrolled diseases) or to compare these findings with salivary flow rate or saliva properties. Also, it would be interesting to longitudinally monitor the healthy subjects with parotid grey scale US modifications in order to evaluate the possible development of diabetes.

In conclusion our study describes a complex US and 2D-SWE evaluation of salivary and lacrimal glands in healthy people and patients with diabetes mellitus and/ or obesity. The main differences between the groups are suggested by the echogenicity, homogeneity, posterior border and the size of glandular area (grey scale criteria). No significant difference of elasticity modulus was found in these groups.

Acknowledgements: We would like to thank to Mr. George Dobre from Pondera Medical that supported our study with the US machine and gave technological advices.

\section{Conflict of interest: none}

\section{Bibliography:}

1. Burke CJ, Thomas RH, Howlett D. Imaging the major salivary glands. Br J Oral Maxillofac Surg 2011;49:261-269. 
2. Howlett DC, Kesse KW, Hughes DV, Sallomi DF. The Role of Imaging in the Evaluation of Parotid Disease. Clin Radiol 2002;57:692-701.

3. Bialek EJ, Jakubowski W, Zajkowski P, Szopinski KT, Osmolski A. US of the major salivary glands: anatomy and spatial relationships, pathologic conditions, and pitfalls. Radiographics 2006;26:745-763.

4. Mansour N, Hofauer B, Knopf A. Ultrasound Elastography in Diffuse and Focal Parotid Gland Lesions. ORL J Otorhinolaryngol Relat Spec 2017;79:54-64.

5. Mantsopoulos K, Klintworth N, Iro H, Bozzato A. Applicability of shear wave elastography of the major salivary glands: values in healthy patients and effects of gender, smoking and pre-compression. Ultrasound Med Biol 2015;41:2310-2318.

6. Badea I, Tamas-Szora A, Chiorean I, et al. Acoustic Radiation Force Impulse quantitative elastography: a new noninvasive technique for the evaluation of parotid glands. A preliminary study in controls and in patients with irradiated nasopharyngeal carcinoma. Med Ultrason 2015;17:308314.

7. Sporea I, Mare R, Lupusoru R, et al. Comparative study between four ultrasound Shear Waves Elastographic methods for liver fibrosis assessment. Med Ultrason 2018;20:265271.

8. Luo J, Cao Y, Nian W, et al. Benefit of Shear-wave Elastography in the differential diagnosis of breast lesion: a diagnostic meta-analysis. Med Ultrason 2018;1:43-49.

9. Bhatia KS, Tong CS, Cho CC, Yuen EH, Lee YY, Ahuja AT. Shear wave elastography of thyroid nodules in routine clinical practice: preliminary observations and utility for detecting malignancy. Eur Radiol 2012;22:2397-2406.

10. Bhatia KS, Cho CC, Tong CS, Lee YY, Yuen EH, Ahuja AT. Shear wave elastography of focal salivary gland lesions: preliminary experience in a routine head and neck US clinic. Eur Radiol 2012;22:957-965.

11. Herman J, Sedlackova Z, Vachutka J, et al. Differential Diagnosis of Parotid Gland Tumors: Role of Shear Wave Elastography. Biomed Res Int 2017;2017:6.

12. Arda K, Ciledag N, Aktas E, Aribas BK, Kose K. Quantitative assessment of normal soft-tissue elasticity using shear-wave ultrasound elastography. AJR Am J Roentgenol 2011;197:532-536.

13. Herman J, Sedlackova Z, Vachutka J, Furst T, Salzman R, Vomacka J. Shear wave elastography parameters of normal soft tissues of the neck. Biomed Pap Med Fac Univ Palacky Olomouc Czech Repub 2017;161:320-325.

14. Caliskan E, Ozturk M, Bayramoglu Z, Comert RG, Adaletli I. Evaluation of parotid glands in healthy children and adolescents using shear wave elastography and superb microvascular imaging. Radiol Med 2018;123:710-718.
15. Giovagnorio F, Pace F, Giorgi A. Sonography of lacrimal glands in Sjogren syndrome. J Ultrasound Med 2000;19:505-509.

16. Scully C, Bagan JV, Eveson JW, Barnard N, Turner FM. Sialosis: 35 cases of persistent parotid swelling from two countries. Br J Oral Maxillofac Surg 2008;46:468-472.

17. Merlo C, Bohl L, Carda C, Gomez de Ferraris ME, Carranza M. Parotid sialosis: morphometrical analysis of the glandular parenchyme and stroma among diabetic and alcoholic patients. J Oral Pathol Med 2010;39:10-15.

18. Orlandi MA, Pistorio V, Guerra PA. Ultrasound in sialadenitis. J Ultrasound 2013;16:3-9.

19. Arya S, Pilania A, Kumar J, Talnia S. Diagnosis of bilateral parotid enlargement (Sialosis) by sonography: A case report and literature review. J Indian Acad Oral Med Radiol 2019;31:79-83

20. Diagnosis and classification of diabetes mellitus. American Diabetes Association. Diabetes Care 2010;33 Suppl 1:S62-S9.

21. Nelson RH. Hyperlipidemia as a risk factor for cardiovascular disease. Prim Care 2013;40:195-211.

22. Purnell JQ. Definitions, Classification, and Epidemiology of Obesity. In: Feingold KR, et al, editors. Endotext. South Dartmouth (MA): MDText.com, Inc.; 2018;2000-.

23. Cornec D, Jousse-Joulin S, Pers JO, et al. Contribution of salivary gland ultrasonography to the diagnosis of Sjogren's syndrome: toward new diagnostic criteria? Arthritis Rheum 2013;65:216-225.

24. Jeong JY, Cho YS, Sohn JH. Role of two-dimensional shear wave elastography in chronic liver diseases: A narrative review. World J Gastroenterol 2018;24:3849-3860.

25. Bouchet P, Gennisson JL, Podda A, Alilet M, Carrié M, Aubry S. Artifacts and Technical Restrictions in 2D Shear Wave Elastography. Ultraschall Med 2018;DOI: 10.1055/a0805-1099.

26. Flores A, Asrani SK. Elastography in Overweight and Obese Patients With Chronic Liver Disease. Clin Gastroenterol Hepatol 2015;13:1510-1512.

27. Wierzbicka M, Kaluzny J, Ruchala M, Stajgis M, Kopec T, Szyfter W. Sonoelastography--a useful adjunct for parotid gland ultrasound assessment in patients suffering from chronic inflammation. Med Sci Monit 2014;20:2311-7.

28. Kaluzny J, Kopec T, Szczepanek-Parulska E, et al. Shear wave elastography: a new noninvasive tool to assess the intensity of fibrosis of irradiated salivary glands in head and neck cancer patients. Biomed Res Int 2014;2014:157809.

29. Katz P, Hartl DM, Guerre A. Clinical ultrasound of the salivary glands. Otolaryngol Clin North Am 2009;42:9731000 ,

30. Dost P, Kaiser S. Ultrasonographic biometry in salivary glands. Ultrasound Med Biol 1997;23:1299-1303. 